\title{
Urinary Bladder Cancer: The Current and Potential Role of MR Imaging in Non-Distant Metastatic Lesions
}

\author{
Huanjun Wang, Yan Guo, Xuhui Zhou, Dong Yang
}

Department of Radiology, The First Affiliated Hospital, Sun Yat-sen University, Guangzhou, China.

Email: dr.guoyan@163.com

Received January $10^{\text {th }}, 2013$; revised February $12^{\text {th }}, 2013$; accepted February $20^{\text {th }}, 2013$

Copyright (C) 2013 Huanjun Wang et al. This is an open access article distributed under the Creative Commons Attribution License, which permits unrestricted use, distribution, and reproduction in any medium, provided the original work is properly cited.

\begin{abstract}
Urinary bladder cancer is the second most common genitourinary malignancy in Westernized countries. A number of emerging imaging techniques have recently been reported in the literature for use in imaging bladder cancer. While the explicit application of MR imaging in bladder cancer especially in non-distant bladder cancer or bladder cancer localized in pelvic remains uncertain and awaits a thorough evaluation. Therefore, this article aims to make a systematic review of previous literature and provide a complete assessment of the value of MR as an evolving imaging tool in localized urinary bladder cancer application. Studies were conducted by searching the MEDLINE and PubMed databases up to 2012 using both medical subject heading (Mesh) and a free text method like "urinary bladder cancer", "magnetic resonance imaging" or "MRI", "diffusion weighted imaging" or "DWI". About 53 articles were selected and analyzed which were considered to be associated with the objective of this systematic review (Table 1). Results showed that MRI especially the newer functional imaging technique like DWI may has a great potential role in localized bladder cancer like early detection, staging, evaluation of tumor aggressiveness and therapeutic responsiveness of patients in the assessment of urinary bladder cancer and so forth. Newer MRI has already proceeded promising results in bladder cancer application; and the explicit role of newer MRI in bladder cancer patients still needs further research and awaits to be thoroughly evaluated.
\end{abstract}

Keywords: Urinary Bladder Cancer; Staging; Progression; Magnetic Resonance Imaging (MRI); Diffusion Weighted Imaging (DWI)

\section{Introduction}

\section{Current Issues in Understanding Bladder Tumors}

It is widely appreciated that urothelial tumors are the most common histopathological type and account for approximately $90 \%-95 \%$ of all bladder tumors [1-3]. This leads one to assume that the diagnosis of bladder cancer is relatively easy or straightforward. However, this overly simplistic consideration, when one considers the multifactorial nature of the management of bladder cancer, actually goes well beyond the diagnosis of the tumor itself. This includes such complex factors as the staging of bladder tumors, the aggressiveness of the specific tumor type and perhaps even therapeutic response assessment of patients presenting with this disease. Further complicating these considerations is the realization of the multifocal nature of bladder tumor, the high recurrence rate, and a certain trend in disease progression. These features collectively convince us that both early screening and preoperative staging are beneficial if an optimal treatment plan is to be realized.

The preoperative estimation for tumor stage is a multifaceted process, requiring a combination of clinical and radiological assessments to objectively evaluate the extent of the tumor growth no matter organ-confined or nonorgan-confined. An accurate preoperative staging of disease is the key to assuring a correct treatment regimen, and in this regard, prognosis largely depends on clinical and radiological staging at presentation [4].

Pathologically, bladder cancers are staged using a TNM staging system. And according to the 2004 World Health Organization/International Society of Urological Pathology classification, a two-tiered grading system for bladder cancer was introduced, which categorized bladder tumors as low and high grade, additionally, it was classified as belonging to either the noninvasive or invasive type of tumor. Tumors with a high frequency and 
Table 1. A general summary for the 53 articles associated with this review which is mainly about bladder cancer MR imaging.

\begin{tabular}{|c|c|c|c|c|c|}
\hline \multirow{2}{*}{ Mype } & \multirow{2}{*}{ Review } & \multicolumn{3}{|c|}{ Original article } & \multirow{2}{*}{ Tota } \\
\hline & & DWI & DCE & others & \\
\hline Systematic & 12 & - & 1 & 1 & 14 \\
\hline $\begin{array}{l}\text { Diagnosis/ } \\
\text { detection }\end{array}$ & - & 4 & 1 & 3 & 8 \\
\hline Staging/grade & 2 & 4 & 8 & 7 & 21 \\
\hline Lymph nodes & - & 3 & - & - & 3 \\
\hline $\begin{array}{l}\text { Therapeutic } \\
\text { evaluation }\end{array}$ & - & 3 & - & - & 3 \\
\hline Pathology & - & - & - & 4 & 4 \\
\hline Total & 14 & 14 & 10 & 15 & 53 \\
\hline
\end{tabular}

"_" indicate that there's no article on that aspect. "Systematic" represents that the article about bladder cancer MR imaging is a comprehensive article involve many aspects about bladder cancer. "Diagnosis/detection" represents that the article is mainly about the diagnosis or detection of bladder cancer. "Staging/grade" represents that the article is mainly about the bladder cancer staging or tumor grade. "Lymph nodes" indicates that the article is subjected to the lymphatic metastasis associated with bladder cancer. "Therapeutic evaluation" represents that the article is about the therapy response evaluation for bladder cancer patients with MR imaging. "Pathology" represents that the article is about the pathology of bladder cancer related to bladder cancer MR imaging. "others" indicates those articles do not subject to the content about DWI (diffusion weighted MR imaging) or DCE (dynamic contrast enhanced MR imaging).

exhibiting highly varied biologic behavior were classified as high-grade tumors, and those unlikely to present aggressive behavior were defined as low-grade tumors [5]. We define those bladder cancers localized in pelvic which contain those tumors belonging to or lower than $\mathrm{T} 3$ as well as those tumors staging in T4 without distant metastasis no matter lymphatic or distant organ metastasis as non-distant bladder cancer lesions.

In general, surgical treatment of patients with early stage tumors like $\mathrm{T} 1$, or lower stage bladder tumors are achieved by an initial transurethral resection (TUR) procedure. Those presenting with a T2 or higher stage with organ-confined tumors usually undergo a partial or radical cystectomy, with or without adjuvant therapies $[1,2]$.

While there's a fact we have to admitted is that there's a proportion of lower stage or non-muscle invasive bladder tumors remaining in a high grade state, and almost $50 \%$ of non-muscle invasive lesions will recur, among which, 30\% will experience disease progression into muscle-invasive lesions [6]. Management options for such high-grade noninvasive tumors might include radical cystectomy or intravesical chemotherapy.

However, there remains no reliable mean to predict which high-grade non-muscle invasive cancers will pro- gress to an invasive form or even display subsequent metastases. If all patients with high-grade noninvasive tumors underwent the therapeutic options as mentioned above, we submit that it would highly likely constitute an over-treatment of those patients by exposing them to the undue additional risk of complications and associated toxicity. This would of course reduce the quality of patients' life, especially for those who likely have no realistic expectation or potential for disease progression [7]. Thus, development of new prognostic markers that may be predictive of tumor behavior would be more useful in the context of patient counseling and treatment selection [8].

Moreover, it is important to mention that $20 \%-25 \%$ of epithelial tumors are muscle invasive and are often of the high histologic grade [9]. Similarly, up to $50 \%$ of these muscle-invasive epithelial tumors will progress with a high likelihood of death, despite such adequate treatment approaches like radical cystectomy and pelvic lymph node dissection [10-12]. Unfortunately, there is no reliable tool that can predict which of the patients with high-grade tumor will likely go on to develop metastasis. Therefore, these uncertainties present many challenges in disease management, especially given the need to decide the most effective therapeutic option.

\section{Application of Imaging in Bladder Tumors}

Since the depth of tumor invasion, and estimation of metastatic condition especially for non-organ-confined bladder tumors cannot be reliably determined by cystoscopy, bladder imaging provides a vital tool in the disease management protocol. One of the most promising imaging tools that has been studied most recently is the highly developed and advanced magnetic resonance imaging (MRI) technique with a high magnetic field strength of 3.0 Tesla $(3.0 \mathrm{~T})$. Thus in the following sections, we aim to mainly discuss the current uses and potential role of MRI in the diagnosis and management of bladder tumors especially those non-distant metastatic lesions, with particular emphasis on the role provided by the 3.0 T MRI.

\subsection{MRI Techniques}

As compared other imaging techniques like ultrasound and CT, MRI has many advantages. These include no risk of radiation exposure and subsequent harmful effects, having an intrinsic high soft tissue resolution, and being able to provide multi-planar parameters and imaging capabilities, among others. Currently, the most commonly used scanners for MRI of the bladder are equipped with a $1.5 \mathrm{~T}$ magnetic scanner. Sequences are typically as follows: a large field of view (FOV) of T1-weighted (T1W) 
imaging on which bladder cancers often appears with an intermediate signal intensity similar to muscle tissue, which actually assists in the identification of infiltration of the perivesical fat by the tumor, lymphadenopathy and bone metastasis.

High resolution fast T2-weighted (T2W) imaging can be obtained with a small FOV and a large matrix on which the bladder cancer manifests as a low signal intensity, providing information on tumor depth and involvement of adjacent structures [13-16]. This is possible because the normal bladder muscle wall manifests itself one or two layers of low (low inner and high outer) signal intensity on T2WI images [17]. Just as image (Figure $\mathbf{1}(\mathrm{A})$ ) which is a high resolution fast T2-weighted image with non-fat suppression obtaining in our department shows, the tumor presents a hypointense signal with an intact low signal bladder muscle wall. In addition, the use of T1-weighted fast spoiled gradient-echo for dynamic contrast-enhanced (DCE) images is often obtained within $90 \mathrm{~s}$ after contrast injection. These images are used to optimize cancer-bladder wall contrast [18]. Bladder cancer involving the mucosa and sub-mucosa often shows an early (about $20 \mathrm{~s}$ after contrast injection) and prominent enhancement compared with the uninvolved bladder wall which enhance late, usually $60 \mathrm{~s}$ after the contrast injection $[13,15,19]$. And the image (Figure 1(B)) is a dynamic contrast enhanced image obtaining from the same patient as (Figure 1(A)) in our department, which shows that the tumor presenting an early significant enhancement with an intact bladder muscle wall remaining to be low signal.

The application of diffusion weighted MR (DWI), with 3.0 T MR scanner in bladder cancer is a relatively new and evolving technique in recent years. The basic principal of DWI is that it quantifies the diffusion of water molecules in tissues. DWI images thus provide both
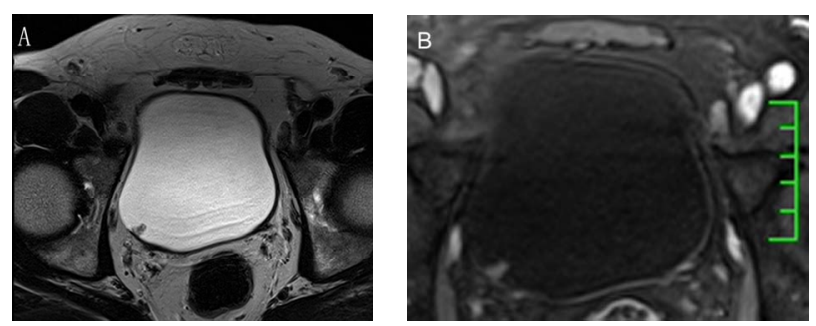

Figure 1. A 51-year-old male with a 1-week history of gross hematuria. A tumor lesion localized in the right posterior bladder wall was detected and showed a low SI which contrasted with the high SI of urine. The bladder wall underlying the tumor base can be seen as an intact low SI (A). On DCE images, the lesion presented as an early enhancement, and the bladder wall under the tumor base also manifested as an intact structure (B). The patient shown here has undergone a transurethral resection and confirmed to have been diagnosed with a non-invasive low-grade papillary urothelial carcinoma. qualitative and quantitative information, which can be applied in the differentiation of benign from malignant tissue without implementing intravenous contrast material [20,21]. Currently, DWI images are usually obtained with a single-shot SE echo planar sequence performed with a chemical shift selective fat-suppression techniques with $b$ values of 0 and 800 , or $1000 \mathrm{sec} / \mathrm{mm}^{2}$, with diffusion gradients being applied in three orthogonal directions. Routinely, images are obtained in the axial planes, and sometimes other institutions will obtain images taken in the sagittal planes.

On DWI images, like other malignancies ,bladder tumor lesions often can be detected by visual assessment as a high-SI lesion on high-b-value images (e.g., 800 - 1000 $\mathrm{sec} / \mathrm{mm}^{2}$ ) even it is small partly because the diffusion of water molecule is restricted, which results from greater cellularity, decrease of the extracellular space as well as the tissue organization distortion. This clearly demonstrates an obvious contrast with the low SI of urine in the full bladder and in terms of the surrounding fat. The bladder wall will usually present as an intermediate SI on high b-value images just as image (Figure 2(A)) shows which is obtained from a bladder cancer patient referring to our hospital. By contrast, on the corresponding ADC map, the tumor often presents as an obvious low intensity signal producing a contrast with high SI urine and peripheral structures, like the image (Figure 2(B)) showing, which is a corresponding ADC map with previous image (Figure 2(A)).

\subsection{Bladder Cancer Staging by MRI}

MRI especially the recently emerging new DWI-MRI technique have been reported can have clinical utility at all stages of a bladder cancer patient's journey from detection to diagnosis, for staging and assessing therapy
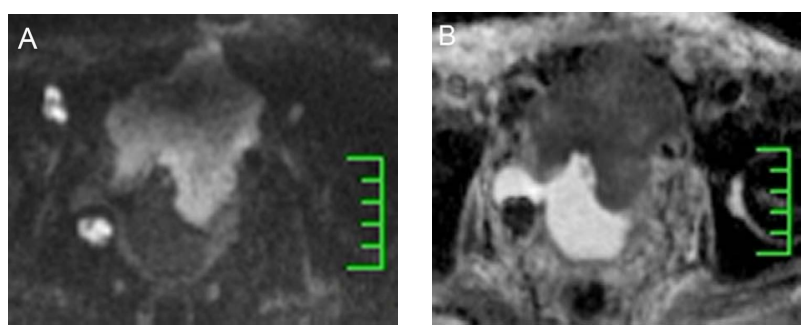

Figure 2. A 74-year-old male presenting with bladder cancer had undergone a transurethral resection seven years prior. In addition, the tumor recurred and progressed. A DWI image is shown in which the tumor lesion shows an obvious extensive high SI to the anterior abdominal wall which produced a contrast with the low SI of urine (A). However, a diverticulum was detected on the right posterior bladder wall, inside of which a high SI was identified, and showed another tumor lesion. The corresponding ADC map is also shown (B), where the tumor lesion manifested a low SI which was contrary to those shown on DWI image. 
response, and finally, for assessing relapse, though questions still revolve around whether the reported results can be measured reliably and reproducibly and whether the results predict important clinical outcomes related to therapy [20]. And in bladder cancer, patients usually undergo bladder MRI is not used for tumor diagnosis per se, since patients have usually already been examined by ultrasound, CT or cystoscopy prior to their MRI scan, and most times have already been diagnosed as having bladder tumors. Though there's no guidelines confirmed to be as a standard yet, MRI is often being used to characterize extravesical growth patterns of the tumor, to guide neoadjuvant therapy, to refine surgical approaches, to monitor response and relapse after various therapeutic regimes and to assist in predicting and planning for the complications of advancing disease.

\subsubsection{Staging Criteria on MR}

\section{1) T2WI imaging}

The current anatomical staging criteria commonly used for T2WI images are as follows: a lesion with intact low signal intensity (SI) muscle-layer at the base of the tumor, is diagnosed as T1 or less (NMIBC); a lesion with focally disrupted low signal muscle-layer without perivesical infiltration, is diagnosed as T2 (MIBC). In addition, a lesion extending into the perivesical fat (T3) or the adjacent organs (T4) is also diagnosed as MIBC [22-24].

\subsubsection{Dynamic contrast enhancement (DCE)}

On MRI sequence images, sub-mucosal linear enhancement (SLE) is depicted immediately after the injection of contrast agent, and the signal intensity of the muscle layer remains low. Therefore, an intact SLE adjacent to a tumor is thought to be indicative of stage T1 or lower. When the SLE is disrupted by a tumor in the absence of tumor infiltration into the perivesical fat, then stage $\mathrm{T} 2$ is considered. The criteria for considering stages T3 and T4 are remarkably similar to those shown for T2WI images $[16,25,26]$.

\subsubsection{Diffuse Weighted Imaging (DWI)}

Currently, most studies describing bladder cancer staging using DWI, applied the criteria first proposed by Takeuchi et al. proposed [27]. Using this system a thin, flat, high SI area corresponding to the tumor indicates stage T1 or lower. Similarly, a high SI tumor with a low SI submucosal stalk or a thickened sub-mucosa indicates stage T1 or lower. By contrast, a high SI tumor without a submucosal stalk, and with a smooth tumor margin, indicates stage T2. Additionally, extension of the tumor into the perivesical fat with an irregular margin indicates stage $\mathrm{T} 3$, and extension into adjacent organs indicates stage T4.

\subsection{Initial Results for Bladder Tumor Staging}

Using conventional MRI, previous studies have demonstrated a staging accuracy which ranged between $72 \%$ and $95 \%$. However, these studies were performed with low field-strength scanners (0.5 Tesla) and contained a small sample size [22,24,28]. In addition, Buy et al. reported a staging accuracy of $60 \%$ for non-enhanced MR images [23], and in this study under-staging (at a rate of $33 \%$ ) was the most common error. Tekes et al. [16] reported that $81 \%$ of bladder tumors showed a similar SI for the bladder muscle wall on T2WI imaging. Furthermore, the most common error found for bladder cancer in T2WI images, was over-staging due to an insufficient signal contrast. However, Tekes et al. confirmed that the accuracy of dynamic-contrast-enhanced MRI (DCE MRI) was highest when differentiating between the non-muscle invasive as compared the muscle invasive (85\%) lesions and when comparing the non-organ confined to the organconfined disease $(82 \%)$. These observations were concordant to those of Tillou et al. [29], which contained sixty patients, a larger sample size than mentioned above.

The value of DCE-MRI in bladder cancer may have find clinical utility in evaluating enhancement patterns of bladder lesions $[4,30]$. However, this idea remains highly controversial. SLE is considered to be major signal marker for the differentiation of $\mathrm{T} 1$ or lower versus $\mathrm{T} 2$ or higher stages. However, at the current time, it would be premature to draw firm conclusions on whether or not the use of gadolinium $(\mathrm{Gd})$-enhanced MRI has a supplementary value in diagnosing bladder cancer. Others have shown that Gd-DTPA enhancement in MRI, improved the accuracy of cancer staging in intramural bladder cancer [31], and others also report that with the use of Gdenhanced images, the sensitivity of detecting bladder cancer and the accuracy of staging of non-muscle invasive bladder tumors has been improved [32,33]. By contrast, Rajesh reported that this approach provided additional staging information for bladder cancer, but only in $3 \%$ of patients and concluded that routine use of Gd-enhanced images was not required [34].

When combined with the DWI approach, a study by Abou-El-Ghar [35] of 106 bladder cancer patients showed a promising result. One of the major conclusions of this study was that the diagnostic performance of DW MR imaging in the identification of bladder tumors exhibited a sensitivity of $98.1 \%$ (104 of 106), a specificity of $92.3 \%$ (24 of 26), and a positive predictive value (PPV) of $100 \%$ (104 of 104). In addition, DWI scanning enabled detection of 121 of the 123 tumors noted by conventional cystoscopy. In this analysis, only two false negatives with diameters of $3 \mathrm{~mm}$ and $4 \mathrm{~mm}$ respectively were identified [35]. In another study comprising 40 patients presenting with 52 bladder tumors, it was shown 
that the overall accuracy of T-stage diagnosis had increased from $67 \%$ with the use of only T2-weighted images to $88 \%$ when $\mathrm{T} 2$-weighted images were combined with DW images [27]. This observation was marginally superior as compared with T2-weighted and DCE images, where in an accuracy of $79 \%$ was reported. In a study of only 18 patients, Watanabe also reported that with the use of DWI, the over-staging rate had been reduced to $5 \%$ as compared with a rate of $16 \%$ with DCE imaging [36].

In addition, the measurement of $\mathrm{ADC}$ values from $\mathrm{DW}$ imaging is currently considered the optimal imaging method for in vivo quantification of the combined effects of capillary perfusion and water diffusion. Moreover, on the corresponding ADC maps, the bladder tumors display a low SI, which is in contrast to the high SI seen with urine. This approach enables delineation of the tumor and quantification by means of the ADC value, which is usually around $1 \times 10^{-3} \mathrm{~mm}^{2} / \mathrm{sec}$ in a malignant tumor, if $\mathrm{b}$ values of 0 and $800-1000 \mathrm{sec} / \mathrm{mm}^{2}$ are applied [24,27].

In general, the absolute $\mathrm{ADC}$ values for tumors are significantly lower than those found for peripheral structures, which is markedly indicative that a malignant lesion exists. However, some inconsistent results still exist according to several prior studies. For example, Matsuki et al. reported that there was no overlap between the ADC values of the tumors and those of the urine or bladder wall. However, overlap could be seen between tumors and the prostate or seminal vesicles [37]. In another study, comprising of 43 patients, it was shown that although there was no overlap between the ADC values of the tumors and the urine, overlaps between tumors and the bladder wall, prostate, or seminal vesicles exist [38]. The inconsistency may due to the fact that there is substantial interviewer variation of the ADC value for any particular region of interest in a relatively thin bladder wall [39]; moreover, the ADC value may be difficult to reproduce since it can alter between systems depending on many variables including the coil system, the vendor, the imaging protocol and others. It may be affected by many factors associated with variability in the subject's tissue itself, and this would include such variables as body temperature, tissue pressure, perfusion rate or the magnetic environment of each individual, and so on [40, 41].

\section{Local Lymphnode Metastasis}

The condition of regional lymph node metastasis is also an essential determinant of survival for patients, and this is despite effective local and systematic therapy [10-12]. Prior studies have shown that extended lymphadenectomy is substantiated by the fact that $33 \%$ of patients with unexpected microscopic nodal involvement at the time of cystectomy have metastatic lesions [42]. There- fore, preoperative assessment of nodal status is crucial though it is not wholly conclusive.

The diagnosis of benign or metastatic lymph nodes using conventional methods like CT or MRI are based on lymph node appearance such as size, contour and internal contrast enhancement, among other factors. Usually, pelvic nodes greater than $8 \mathrm{~mm}$ and abdominal nodes greater than $10 \mathrm{~mm}$ in the maximal short axis diameter are considered metastatic $[43,44]$. Despite these criteria, there remains a lack of both sensitivity and specificity, which often leads to a high false negative result. The major reason accounting for this is that small metastatic lymph node may be missed. Additionally, marginal locations of reactive enlarged benign lymph nodes are also often misdiagnosed. Although there is some evidence that conventional MRI is superior to CT scanning in detecting sub-centimeter lymph nodes, MRI still can not distinguish those lymph nodes with micro-metastatic disease $[45,46]$.

Previously, the use of ferumoxtran-10-enhanced MR imaging was reported. Using this approach, there was significant improvement in nodal staging in patients with bladder cancer by detection of metastases even in normal-sized lymph nodes. In addition, the sensitivity and negative predictive values significantly improved nodal staging at post-contrast (from $76 \%$ to $96 \%$ ) as compared with those at pre-contrast imaging (from $91 \%$ to $98 \%$ ). However, the limitation associated with this approach is that the time period to complete the examination is extended to some degree [47]. Another study conducted by Thoeny et al. used an approach of combining USPIO and DW-MRI (USPIO-DW-MRI) in the detection of pelvic lymph node metastases. According to this approach, lymph node signal intensity is contrasted with that of the "classic" reading method that is applied in the evaluation of USPIO images with and without DW-MRI. This study showed that 24 lymph nodes out of 26 (92\%) were correctly diagnosed as positive by USPIO-DW-MRI, and only two metastasis $(1.0 \times 0.2 \mathrm{~mm} ; 0.7 \times 0.4 \mathrm{~mm}$ respectively) were misdiagnosed. This demonstrated that USPIO-DW-MRI can significantly improve the accuracy of detecting lymph node metastasis in pelvic malignancies, even in normal-sized nodes. Despite this, the limitation of this technique was concordant with previous studies, in which an extended period was required for image acquisition. That having been said, the USOIO approach is not currently widespread in clinical practice [48].

While DWI as a functional MRI imaging technique, combining the measurement of ADC values, it represents a interesting field of research and this technique holds much promise for the future. A study that assessed the application of DWI in the detection of pelvic lymph node metastases in bladder cancer patients showed that the $\mathrm{ADC}$ value of metastatic lymph nodes was significantly 
lower than that of non-metastatic lymph nodes. In addition, the $\mathrm{ADC}$ value of $0.86 \times 10^{-3} \mathrm{~mm}^{2} / \mathrm{s}$ provided the clearest separation between metastatic and non-metastatic lymph nodes according to the ROC curve described in this study [49].

However, although MRI has exhibited many advantages in the application of non-distant metastatic lesions for bladder cancer patients, we cannot deny the limitation still exist given the distant metastatic lesions are unascertainable especially when the metastasis are diffused, on which, ${ }^{18} \mathrm{FDG}-\mathrm{PET} / \mathrm{CT}$ is much more sensitive in those extrapelvic metastasis detection.

\section{Evaluation of Tumor Aggressiveness}

Conventional MRI has been rarely used in differentiating bladder cancer by histological grade. While the widespread application of functional DWI-MR in other organ systems like brain, breast, rectum, etc, has proven to be useful, it has also been consistently used recently in the examination of the urogenital system. Takeuchi et al. used the ADC map to generate a region of interest that was larger than $5 \mathrm{~mm}$ in bladder tumors to evaluate the degree of diffusion in the tumor. In this study, a correlation was made between the ADC values and histopathologic findings of the tumor, and this showed that the mean ADC value (b values 0 and $1000 \mathrm{sec} / \mathrm{mm}^{2}$ ) of G3 bladder tumors $\left(0.81 \pm 0.11 \times 10^{-3} \mathrm{~mm}^{2} / \mathrm{s}\right)$ was significantly lower than that of G1 and G2 tumors. However, there was a significant overlap between G1 and G2 tumors, for which the ADC values were respectively measured as $(1.29 \pm 0.21) \times 10^{-3} \mathrm{~mm}^{2} / \mathrm{s},(1.13 \pm 0.24) \times$ $10^{-3} \mathrm{~mm}^{2} / \mathrm{s}$, and one of the key reasons for this was that these studies referred to the 1973 WHO classification for tumor grading $[27,50]$.

Additionally, Rosenkrantz reported on a study comprised of 17 patients with pathological confirmation of high-grade bladder cancers. This study showed that the ADC value was significantly lower among cases with metastatic disease than those in the absence of metastatic disease with an ADC value of $1.07 \pm 0.18 \times 10^{-3} \mathrm{~mm}^{2} / \mathrm{s}$ in the former and $1.45 \pm 0.22 \times 10^{-3} \mathrm{~mm}^{2} / \mathrm{s}$ in the latter. In addition, the sensitivity and specificity were $87.5 \%$ and $100 \%$ respectively, which suggested that ADC value may serve as a supplement parameter for predicting tumor behavior and making appropriate treatment decisions.

\section{Therapeutic Response Evaluation and Postresection Follow Up}

Although radical cystectomy is the reference standard in the management of organ-confined muscle-invasive bladder cancer, a proportion of patients undergoing bladdersparing transurethral resection supplemented with radio- therapy or chemotherapy, etc, may achieve a promising outcome and in time, an improvement in their quality of life [51-54]. Usually, only those patients who achieve a complete response after chemotherapy are selected for a bladder-sparing strategy, which is accompanied by additional radiotherapy, otherwise, a radical cystectomy has to be considered. Thus, an accurate treatment response evaluation and prediction of an optimal selection of patients suitable for a bladder-sparing protocol are more necessary $[53,54]$.

We suggest that MRI is the most promising technique, and has the potential to assist in bladder-sparing protocols. Comparable to CT scanning, the conventional T2WI and dynamic enhancement images still present difficulties in distinguishing the residual tumor tissues and the bladder wall thickening, as well as inflammation and fibrotic changes, especially after TUR therapy several weeks after cessation of therapy [55-57]. A recent studies attempting to evaluate the DWI application role in the follow-up of non-muscle invasive urinary bladder carcinoma after transurethral resection concluded that DWMRI has a high reliability in differentiating post-TUR inflammatory changes from bladder tumors recurrence, which is similar to that of cystoscopy [58]. Also DWI appears to possess the potential to monitor the reaction of bladder tumors during the course of treatment. In support of this viewpoint, was a study which demonstrated the application of DWI imaging of bladder tumors, with the major objective of assessing the value of DWI on the therapeutic response of tumors following chemotherapy. The study comprised 20 patients with muscle-invasive bladder cancers which were analyzed by DWI during the therapeutic response of the tumors to induction chemotherapy and radiotherapy. This study reported a higher specificity and accuracy ( $92 \%$ and $80 \%)$ of DWI imaging as compared with T2-weighted MR imaging (45\% and $44 \%$ ) and DCE MRI imaging (18\% and 33\%). Predictably, this approach avoids the issue of misdiagnosis of local edema, fibrosis and inflammation since the residual tumor or recurrence of the tumor according to the latter two me- thods can often lead to an overestimation [59]. Therefore, this study suggested that DWI, as a non-invasive MRI imaging tool may be useful in accurately predicting the pathological response of bladder tumors to induction chemoradiotherapy targeted against MIBC.

\section{Conclusion}

Although most studies were conducted in relatively small cohorts of patients, the study results describing the application of MRI especially DWI, in non-distant metastatic bladder cancer diagnosis staging and prediction of tumor aggression, even in the context of therapeutic response evaluation and postresection follow up, were very 
promising. Therefore, future studies will likely include larger patient cohorts to fully validate the role of newly emerging MRI technique. This is especially true in terms of the use of DWI, which combining the quantitive measurement of the ADC value as a biomarker for accurate $\mathrm{T}$ staging and in the pretreatment of predicted tumor aggression, and perhaps also to evaluate the therapeutic response in bladder cancer patients.

\section{REFERENCES}

[1] A. Saad, D. C. Hanbury, T. A. McNicholas, et al., "A Study Comparing Various Noninvasive Methods of Detecting Bladder Cancer in Urine," BJUI International, Vol. 89, No. 4, 2002, pp. 369-373.

doi:10.1046/j.1464-4096.2001.01699.x

[2] C. A. Sadow, S. G. Silverman, M. P. O'Leary, et al., "Bladder Cancer Detection with CT Urography in an Academic Medical Center," Radiology, Vol. 249, No. 1, 2008, pp. 195-202. doi:10.1148/radiol.2491071860

[3] J. I. Epstein, "The New World Health Organization/International Society of Urological Pathology (WHO/ISUP) Classification for TA, T1 Bladder Tumors: Is It an Improvement?" Critical Reviews in Oncology/Hematology, Vol. 47, No. 2, 2003, pp. 83-89.

[4] M. A. Amendola, G. M. Glazer, H. B. Grossman, et al., "Staging of Bladder Carcinoma: MRI-CT-Surgical Correlation," American Journal of Roentgenology, Vol. 146, No. 6, 1986, pp. 1179-1183. doi:10.2214/ajr.146.6.1179

[5] W. Otto, S. Denzinger, H. M. Fritsche, et al., "The WHO Classification of 1973 Is More Suitable than the WHO Classification of 2004 for Predicting Survival in pT1 Urothelial Bladder Cancer," BJUI International, Vol. 107, No. 3, 2011, pp. 404-408. doi:10.1111/j.1464-410X.2010.09515.x

[6] B. W. van Rhijn, M. Burger, Y. Lotan, et al., "Recurrence and Progression of Disease in Non-Muscle-Invasive Bladder Cancer: From Epidemiology to Treatment Strategy," European Urology, Vol. 56, No. 3, 2009, pp. 430-442. doi:10.1016/j.eururo.2009.06.028

[7] H. A. Frazier, J. E. Robertson and D. F. Paulson, "Complications of Radical Cystectomy and Urinary Diversion: A Retrospective Review of 675 Cases in 2 Decades," Journal of Urology, Vol. 148, No. 5, 1992, pp. 14011405.

[8] C. T. Nguyen, A. J. Stephenson and M. W. Kattan, "Are Nomograms Needed in the Management of Bladder Cancer?” Urologic Oncology, Vol. 28, No. 1, 2010, pp. 102107.

[9] C. L. Pashos, M. F. Botteman, B. L. Laskin, et al., "Bladder Cancer: Epidemiology, Diagnosis, and Management," Cancer Practice, Vol. 10, No. 6, 2002, pp. 311-322. doi:10.1046/j.1523-5394.2002.106011.x

[10] J. P. Stein, G. Lieskovsky, R. Cote, et al., "Radical Cystectomy in the Treatment of Invasive Bladder Cancer: LongTerm Results in 1054 Patients," Journal of Clinical Oncology, Vol. 19, No. 3, 2001, pp. 666-675.

[11] S. Madersbacher, W. Hochreiter, F. Burkhard, et al., "Ra- dical Cystectomy for Bladder Cancer Today-A Homogeneous Series without Neoadjuvant Therapy," Journal of Clinical Oncology, Vol. 21, No. 4, 2003, pp. 690-696. doi:10.1200/JCO.2003.05.101

[12] S. F. Shariat, P. I. Karakiewicz, G. S. Palapattu, et al., "Outcomes of Radical Cystectomy for Transitional Cell Carcinoma of the Bladder: A Contemporary Series from the Bladder Cancer Research Consortium," Journal of Urology, Vol. 176, No. 6, 2006, pp. 2414-2422.

[13] J. J. Wong-You-Cheong, P. J. Woodward, M. A. Manning, et al., "From the Archives of the AFIP: Neoplasms of the Urinary Bladder: Radiologic-Pathologic Correlation," Radiographics, Vol. 26, No. 2, 2006, pp. 553-580. doi:10.1148/rg.262055172

[14] M. K. Dighe, P. Bhargava and J. Wright, "Urinary Bladder Masses: Techniques, Imaging Spectrum, and Staging," Journal of Computer Assisted Tomography, Vol. 35, No. 4, 2011, pp. 411-424. doi:10.1097/RCT.0b013e31821c2e9d

[15] J. Zhang, S. Gerst, R. A. Lefkowitz, et al., "Imaging of Bladder Cancer," Radiologic Clinics of North America, Vol. 45, No. 1, 2007, pp. 183-205. doi:10.1016/j.rcl.2006.10.005

[16] A. Tekes, I. Kamel, K. Imam, et al., "Dynamic MRI of Bladder Cancer: Evaluation of Staging Accuracy," American Journal of Roentgenology, Vol. 184, No. 1, 2005, pp. 121-127. doi:10.2214/ajr.184.1.01840121

[17] Y. Narumi, T. Kadota, E. Inoue, et al., "Bladder Wall Morphology: In Vitro MR Imaging-Histopathologic Correlation," Radiology, Vol. 187, No. 1, 1993, pp. 151-155.

[18] S. Venz, J. Ilg, T. Ebert, et al., "Determining the Depth of Infiltration in Urinary Bladder Carcinoma with Contrast Medium Enhanced Dynamic Magnetic Resonance Tomography. With Reference to Postoperative Findings and Inflammation," Urologe A, Vol. 35, No. 4, 1996, pp. 297304.

[19] J. O. Barentsz, G. J. Jager, P. B. van Vierzen, et al., "Staging Urinary Bladder Cancer after Transurethral Biopsy: Value of Fast Dynamic Contrast-Enhanced MR Imaging," Radiology, Vol. 201, No. 1, 1996, pp. 185-193.

[20] A. R. Padhani, G. Liu, D. M. Koh, et al., "DiffusionWeighted Magnetic Resonance Imaging as a Cancer Biomarker: Consensus and Recommendations," Neoplasia, Vol. 11, No. 2, 2009, pp. 102-125.

[21] D. M. Koh and D. J. Collins, "Diffusion-Weighted MRI in the Body: Applications and Challenges in Oncology," American Journal of Roentgenology, Vol. 188, No. 6, 2007, pp. 1622-1635. doi:10.2214/AJR.06.1403

[22] M. R. Fisher, H. Hricak and E. A. Tanagho, "Urinary Bladder MR Imaging, Part II, Neoplasm,” Radiology, Vol. 157, No. 2, 1985, pp. 471-477.

[23] J. N. Buy, A. A. Moss, C. Guinet, et al., "MR Staging of Bladder Carcinoma: Correlation with Pathologic Findings," Radiology, Vol. 169, No. 3, 1988, pp. 695-700.

[24] K. S. Rholl, J. K. Lee, J. P. Heiken, et al., "Primary Bladder Carcinoma: Evaluation with MR Imaging," Radiology, Vol. 163, No. 1, 1987, pp. 117-121.

[25] N. Hayashi, H. Tochigi, T. Shiraishi, et al., "A New Stag- 
ing Criterion for Bladder Carcinoma Using GadoliniumEnhanced Magnetic Resonance Imaging with an Endorectal Surface Coil: A Comparison with Ultrasonography," BJUI International, Vol. 85, No. 1, 2000, pp. 32-36. doi:10.1046/j.1464-410x.2000.00358.x

[26] B. Kim, R. C. Semelka, S. M. Ascher, et al., "Bladder Tumor Staging: Comparison of Contrast-Enhanced CT, T1- and T2-Weighted MR Imaging, Dynamic Gadolinium-Enhanced Imaging, and Late Gadolinium-Enhanced Imaging," Radiology, Vol. 193, No. 1, 1994, pp. 239-245.

[27] M. Takeuchi, S. Sasaki, M. Ito, et al., "Urinary Bladder Cancer: Diffusion-Weighted MR Imaging-Accuracy for Diagnosing T Stage and Estimating Histologic Grade," Radiology, Vol. 251, No. 1, 2009, pp. 112-121. doi:10.1148/radiol.2511080873

[28] M. A. Amendola, G. M. Glazer, H. B. Grossman, et al., "Staging of Bladder Carcinoma: MRI-CT-Surgical Correlation," American Journal of Roentgenology, Vol. 146. No. 6, 1986, pp. 1179-1183. doi:10.2214/ajr.146.6.1179

[29] X. Tillou, E. Grardel, M. Fourmarier, et al., "Can MRI Be Used to Distinguish between superficial and Invasive Transitional Cell Bladder Cancer?" Progrès en Urologie, Vol. 18, No. 7, 2008, pp. 440-444. doi:10.1016/j.purol.2008.04.019

[30] R. B. Rafal and J. A. Markisz, "Urachal Carcinoma: The Role of Magnetic Resonance Imaging," Urologic Radiology, Vol. 12, No. 4, 1991, pp. 184-187.

[31] A. Tanimoto, Y. Yuasa, Y. Imai, et al., "Bladder Tumor Staging: Comparison of Conventional and GadoliniumEnhanced Dynamic MR Imaging and CT," Radiology, Vol. 185, No. 3, 1992, pp. 741-747.

[32] V. Scattoni, P. L. Da, R. Colombo, et al., "Dynamic Gadolinium-Enhanced Magnetic Resonance Imaging in Staging of Superficial Bladder Cancer," Journal of Urology, Vol. 155, No. 5, 1996, pp. 1594-1599. doi:10.1016/S0022-5347(01)66139-1

[33] J. M. Neuerburg, K. Bohndorf, M. Sohn, et al., "Staging of Urinary Bladder Neoplasms with MR Imaging: Is GdDTPA Helpful?" Journal of Computer Assisted Tomography, Vol. 15, No. 5, 1991, pp. 780-786. doi:10.1097/00004728-199109000-00010

[34] A. Rajesh, H. K. Sokhi, R. Fung, et al., "Bladder Cancer: Evaluation of Staging Accuracy Using Dynamic MRI," Clinical Radiology, Vol. 66, No. 12, 2011, pp. 1140-1145. doi:10.1016/j.crad.2011.05.019

[35] M. E. Abou-El-Ghar, A. El-Assmy, H. F. Refaie, et al., "Bladder Cancer: Diagnosis with Diffusion-Weighted MR Imaging in Patients with Gross Hematuria," Radiology, Vol. 251, No. 2, 2009, pp. 415-421. doi:10.1148/radiol.2503080723

[36] H. Watanabe, M. Kanematsu, H. Kondo, et al., "Preoperative T Staging of Urinary Bladder Cancer: Does Diffusion-Weighted MRI Have Supplementary Value?" American Journal of Roentgenology, Vol. 192, No. 5, 2009, pp. 1361-1366. doi:10.2214/AJR.08.1430

[37] M. Matsuki, Y. Inada, F. Tatsugami, et al., "DiffusionWeighted MR Imaging for Urinary Bladder Carcinoma: Initial Results," European Radiology, Vol. 17, No. 1, 2007, pp. 201-204. doi:10.1007/s00330-006-0281-7
[38] A. El-Assmy, M. E. Abou-El-Ghar, H. F. Refaie, et al., "Diffusion-Weighted MR Imaging in Diagnosis of Superficial and Invasive Urinary Bladder Carcinoma: A Preliminary Prospective Study," Scientific World Journal, Vol. 8, 2008, pp. 364-370. doi:10.1100/tsw.2008.55

[39] D. M. Koh, T. Takahara, Y. Imai, et al., "Practical Aspects of Assessing Tumors Using Clinical DiffusionWeighted Imaging in the Body," Magnetic Resonance in Medical Sciences, Vol. 6, No. 4, 2007, pp. 211-224. doi:10.2463/mrms.6.211

[40] S. Kobayashi, F. Koga, S. Yoshida, et al., "Diagnostic Performance of Diffusion-Weighted Magnetic Resonance Imaging in Bladder Cancer: Potential Utility of Apparent Diffusion Coefficient Values as a Biomarker to Predict Clinical Aggressiveness," European Radiology, Vol. 21, No. 10, 2011, pp. 2178-2186. doi:10.1007/s00330-011-2174-7

[41] J. B. Unger, J. J. Ivy, M. R. Ramaswamy, et al., "WholeBody [18F]Fluoro-2-Deoxyglucose Positron Emission Tomography Scan Staging Prior to Planned Radical Hysterectomy and Pelvic Lymphadenectomy," International Journal of Gynecological Cancer, Vol. 15, No. 6, 2005, pp. 1060-1064. doi:10.1111/j.1525-1438.2005.00262.x

[42] B. H. Bochner, D. Cho, H. W. Herr, et al., "Prospectively Packaged Lymph Node Dissections with Radical Cystectomy: Evaluation of Node Count Variability and Node Mapping," Journal of Urology, Vol. 172, No. 4, 2004, pp. 1286-1290.

[43] J. O. Barentsz, M. R. Engelbrecht, J. A. Witjes, et al., "MR Imaging of the Male Pelvis," European Radiology, Vol. 9, No. 9, 1999, pp. 1722-1736. doi: $10.1007 / \mathrm{s} 003300050916$

[44] R. E. Dorfman, M. B. Alpern, B. H. Gross, et al., "Upper Abdominal Lymph Nodes: Criteria for Normal Size Determined with CT," Radiology, Vol. 180, No. 2, 1991, pp. 319-322.

[45] A. Saokar, T. Islam, M. Jantsch, et al., "Detection of Lymph Nodes in Pelvic Malignancies with Computed Tomography and Magnetic Resonance Imaging," Clinical Imaging, Vol. 34, No. 5, 2010, pp. 361-366. doi:10.1016/j.clinimag.2009.07.004

[46] T. K. Jensen, P. Holt, O. Gerke, et al., "Preoperative Lymph-Node Staging of Invasive Urothelial Bladder Cancer with 18F-Fluorodeoxyglucose Positron Emission Tomography/Computed Axial Tomography and Magnetic Resonance Imaging: Correlation with Histopathology," Scandinavian Journal of Urology, Vol. 45, No. 2, 2011, pp. 122-128. doi:10.3109/00365599.2010.544672

[47] W. M. Deserno, M. G. Harisinghani, M. Taupitz, et al., "Urinary Bladder Cancer: Preoperative Nodal Staging with Ferumoxtran-10-Enhanced MR Imaging," Radiology, Vol. 233, No. 2, 2004, pp. 449-456. doi:10.1148/radiol.2332031111

[48] H. C. Thoeny, M. Triantafyllou, F. D. Birkhaeuser, et al., "Combined Ultrasmall Superparamagnetic Particles of Iron Oxide-Enhanced and Diffusion-Weighted Magnetic Resonance Imaging Reliably Detect Pelvic Lymph Node Metastases in Normal-Sized Nodes of Bladder and Prostate Cancer Patients," European Urology, Vol. 55, No. 4, 
2009, pp. 761-769. doi:10.1016/j.eururo.2008.12.034

[49] R. Papalia, G. Simone, R. Grasso, et al., "Diffusion-Weighted Magnetic Resonance Imaging in Patients Selected for Radical Cystectomy: Detection Rate of Pelvic Lymph Node Metastases," BJUI International, Vol. 109, No. 7, 2012, pp. 1031-1036. doi:10.1111/j.1464-410X.2011.10446.X

[50] R. Montironi and A. Lopez-Beltran, "The 2004 WHO Classification of Bladder Tumors: A Summary and Commentary," International Journal of Surgical Pathology, Vol. 13, No. 2, 2005, pp. 143-153. doi:10.1177/106689690501300203

[51] M. Housset, C. Maulard, Y. Chretien, et al., "Combined Radiation and Chemotherapy for Invasive TransitionalCell Carcinoma of the Bladder: A Prospective Study," Journal of Clinical Oncology, Vol. 11, No. 11, 1993, pp. 21502157.

[52] C. Rodel, C. Weiss and R. Sauer, "Trimodality Treatment and Selective Organ Preservation for Bladder Cancer," Journal of Clinical Oncology, Vol. 24, No. 35, 2006, pp. 5536-5544. doi:10.1200/JCO.2006.07.6729

[53] W. U. Shipley, D. S. Kaufman, E. Zehr, et al., "Selective Bladder Preservation by Combined Modality Protocol Treatment: Long-Term Outcomes of 190 Patients with Invasive Bladder Cancer," Urology, Vol. 60, No. 1, 2002, pp. 62-68.

[54] P. W. Chung, R. G. Bristow, M. F. Milosevic, et al., "LongTerm Outcome of Radiation-Based Conservation Therapy for Invasive Bladder Cancer," Urologic Oncology, Vol. 25, No. 4, 2007, pp. 303-309. doi:10.1016/j.urolonc.2006.09.015

[55] M. J. Dobson, B. M. Carrington, C. D. Collins, et al., "The Assessment of Irradiated Bladder Carcinoma Using Dynamic Contrast-Enhanced MR Imaging," Clinical Radiology, Vol. 56, No. 2, 2001, pp. 94-98. doi:10.1053/crad.2000.0560

[56] P. Therasse, S. G. Arbuck, E. A. Eisenhauer, et al., "New Guidelines to Evaluate the Response to Treatment in Solid Tumors. European Organization for Research and Treatment of Cancer, National Cancer Institute of the United States, National Cancer Institute of Canada," Journal of the National Cancer Institute, Vol. 92, No. 3, 2000, pp. 205-216. doi:10.1093/jnci/92.3.205

[57] R. J. Johnson, B. M. Carrington, J. P. Jenkins, et al., “Accuracy in Staging Carcinoma of the Bladder by Magnetic Resonance Imaging," Clinical Radiology, Vol. 41, No. 4, 1990, pp. 258-263. doi:10.1016/S0009-9260(05)81661-7

[58] A. El-Assmy, M. E. Abou-El-Ghar, H F. Refaie, et al., "Diffusion-Weighted Magnetic Resonance Imaging in Follow-Up of Superficial Urinary Bladder Carcinoma after Transurethral Resection: Initial Experience," BJUI Internationa, Vol. 110, No. 11b, 2012, pp. E622-E627. doi:10.1111/j.1464-410X.2012.11345.x

[59] S. Yoshida, F. Koga, S. Kawakami, et al., "Initial Experience of Diffusion-Weighted Magnetic Resonance Imaging to Assess Therapeutic Response to Induction Chemoradiotherapy against Muscle-Invasive Bladder Cancer," Urology, Vol. 75, No. 2, 2010, pp. 387-391. doi:10.1016/j.urology.2009.06.111 